\title{
Creating epigenetic memory
}

\author{
A three-component system of epigenetic writers and readers preserves \\ memory over many generations of cells.
}

ike many synthetic biologists, Ahmad

(Mo) Khalil from Boston University

describes himself as "really interested in how cellular behavior arises from molecular circuits." But unlike other researchers who have taken a discovery-based approach to find answers, he sought to engineer a system that could establish a certain behavior, in particular a system that retains epigenetic memory: "The philosophy in our lab is that we understand these cellular systems by building them," Khalil explained.

In a project driven by graduate student Minhee Park and jointly supervised by Khalil and Albert Keung at North Carolina State University, the first step was to establish a sequence-specific modification, recruit proteins that could read it, and develop a mechanism of self-propagation without continuous triggers.

The team chose a bacterial DNA adenine methyltransferase as a writer; it methylates $A$ in a GATC motif, a rare modification in metazoan genomes. By fusing the enzyme to a zinc-finger protein that binds a 20-nucleotide synthetic sequence and making the expression of this fusion protein, termed synI, conditional on an inducer molecule, the researchers were able to direct the timing and location of the $\mathrm{m} 6 \mathrm{~A}$ placement. Next they aimed to engineer synthetic reader modules (synR) that translated the m6A marks into transcription of a reporter gene. Their synR combined a bacterial m6A-binding domain with the activation domain of a transcription factor and triggered GFP reporter expression only in response to an $\mathrm{m} 6 \mathrm{~A}$ signal.

One hallmark of an epigenetic network is the spatial spreading of the signal: adding a third construct-one that combined the read and write domains (synRW) - to their synI and synR constructs enabled the researchers to not only establish a mark but also propagate it over genomic distances of several kilobases, where it triggered the expression of the reporter.

To their initial surprise, the activity of the m6A writing enzyme needed to be dampened for this circuit to function. "We naively thought we could put these enzymes together and it will work," recalls Khalil, but they had to select for variants with lower activity. Khalil adds, "Maybe in hindsight it is not terribly surprising. If you look in nature, what you see is that enzymes that are used to write or erase heterochromatin often have lower activity than other enzymes."

This positive propagation feedback loop was the basis for a circuit that established epigenetic memory. A transient dose of the inducer triggered the initial writing of m6A with synI; then the inducer was removed, but the synRW and synR constructs remained active in the cells through many divisions. "We gave a short pulse of the drug to establish the GFP on state," explained Khalil, "and with RW the cells ten generations on remember a drug that is no longer there."

The researchers showed that the epigenetic memory depended on the RW module-without it the reporter signal decays as cells divide-but they also observed that the memory was not as long-lived and stable as that of some naturally occurring epigenetic signals. To increase the stability of the memory, Khalil plans to expand their minimal system of one feedback loop. "The read-write circuit is the core engine, but alone it may be insufficient to establish long-term memory; coupling two distinct feedback loops may help fortify the memory," Khalil reasons. His team is planning to build additional feedback loops onto their circuit.

Synthetic biology straddles the fundamental and applied aspects of science, and Khalil sees a system with sustained memory and output as useful in a therapeutic setting. Although he describes this as a "more futuristic goal," the next steps on the road are obvious, such as thoroughly testing off-targets to prove that the circuit is biologically inert and does not introduce m6A marks where they are not wanted. While $\mathrm{m} 6 \mathrm{~A}$ is rare, it has recently been reported in mouse embryonic stem cells and in human glioblastoma cells; "having tools that can write it and induce it at endogenous sites could be very helpful for some of these studies," concludes Khalil.

\section{Nicole Rusk}

Published online: 30 January 2019

https://doi.org/10.1038/s41592-019-0312-3

\section{Research papers}

Park, M. et al. Engineering epigenetic regulation using synthetic read-write modules. Cell 176, 227-238 (2019).

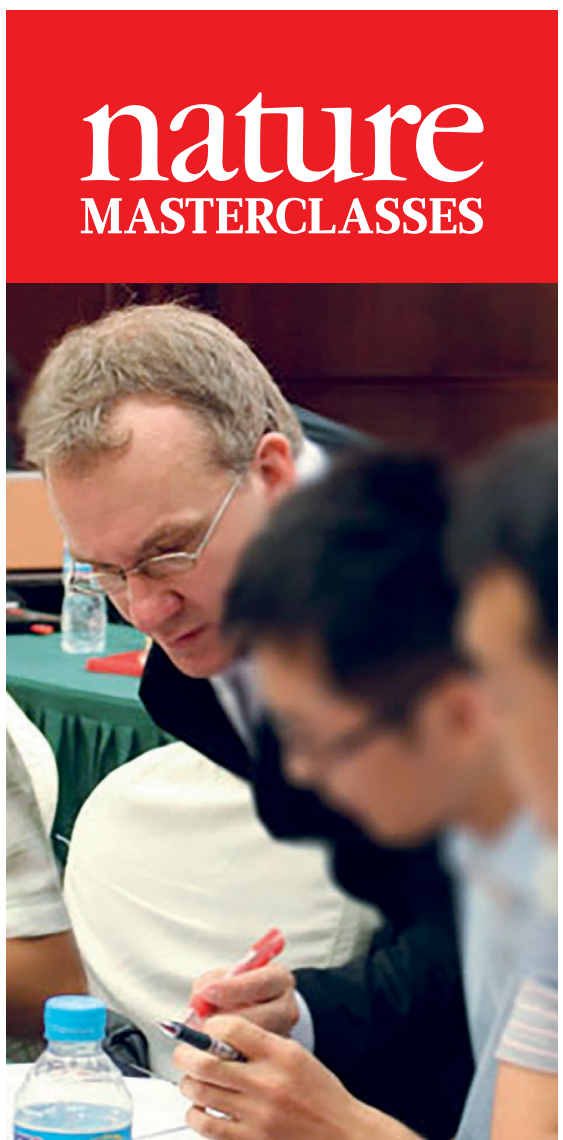

Workshops in

Scientific Writing

and Publishing

Delivered by Nature Research

journal editors and hosted

at institutions worldwide,

researchers learn how to

turn great science into great

papers.

Find out more at masterclasses.nature.com

W masterclasses.nature.com

in Follow us on Linkedln 